\title{
Research on Patent Model Integration Based on Ontology
}

\author{
Liping Zhi \\ Business School, University of Shanghai for Science \& Technology \\ Shanghai 200093, China \\ $\&$ \\ School of Computer Science \& Information Engineering, Anyang Normal University \\ Anyang 455002, China \\ Tel: 86-21-5527-1750Ｅ-mail: 091480029@st.usst.edu.cn
}

Hengshan Wang

Business School, University of Shanghai for Science \& Technology, Shanghai 200093, China

Tel: 86-21-5527-1750 E-mail: 091480029@st.usst.edu.cn

Yazheng Dang

Business School, University of Shanghai for Science \& Technology, Shanghai 200093, China

Tel: 86-21-5527-1750 E-mail: 091480029@st.usst.edu.cn

The research is financed by Shanghai Leading Academic Discipline Project.No.S30504) \& the National Natural Science Foundation of China.No.70901010) \& the Innovation Fund Project For Graduate Student of Shanghai.No.JWCXSL1021).

\begin{abstract}
To integrate models and improve models collaboration, an ontology-based program-logic-combined (PLC) model representation is proposed, which is easily- implemented, reasoning-supported, has high openness and extensibility. Take the TV ontology as an example, OWL is used to describe the semantics of parameters. Based on this semantic match, model integration strategies are designed to create understandable decision problem solutions.
\end{abstract}

Keywords: Decision support system (DSS), Model integration, Model representation, Ontology, OWL

\section{Introduction}

Model is an important part of the decision support system. Along with the development of large decision support system, a growing need for model of integration intelligently and automatically. Nowadays,there are many methods, such as GAMS(H K Bhargava, 1997, P.193-214)proposed by Bhargava, the MIDA posted by Holocher(M Holocher, 1997, P.135-147), KVS(Kaushal Chari, 2003, P.399-413)method proposed by Chari, Byung Kwon packaging decision support module for Web service model(O B Kwon, 2003, P.375-389),but due to defficult to describe the input and output of the model, the existing model semantic properties of integration method is not ideal. How to reuse existing model program and design integration method with high expansibility, openness, automation and intelligent degree, has become an important problem to be solved for the large $I^{3} \mathrm{DSS}$ of the integrated, interactive, intelligent decision support system.

The term "ontology" comes from the field of philosophy that is concerned with the study of being or existence. In 1993, Gruber gave a definition of ontology, namely "ontology is an explicit specification of a conceptualization"(Gruber, 1993, P. 199-220). Then Borst proposed another definition: "ontology is an explicit specification of a shared conceptualization"(Borst, 1997, P.15-19). Studer made deep research based on these two definitions, put forward the current are widely accepted definition: " ontology is a formal, explicit specification of shared conceptual models "(Studer, 1997, P.161-197).Ontology can provide structured language and explicate the relationship between different terms in order that intelligent agent can explain flexibly its meaning without ambiguity (Uschold, 1996, P.93-136). 
OWL (Web Ontology Language) is a W3C (World Wide Web Consortium) recommendatory standard for ontology language, which is a language system based on description logic. OWL can be used to explicitly represent the meaning of terms in vocabularies and the relationship between those terms. OWL facilitates greater machine interpretability of web content than that supported by XML (Extensible Markup Language), RDF (Resource Description Framework), and RDF Schema (RDF-S) by providing additional vocabulary along with a formal semantics. According to the needs of different users, OWL has three increasingly expressive sublanguages: OWL Lite, OWL DL(Description Logics), and OWL Full. To capture domain knowledge from the UML class diagram as much as possible, and to ensure OWL domain ontology completeness and decidability, this paper choose OWL-DL as target language (http://www.w3.org/TR/2004/REC-owl-features-20040210).

It promotes the development of some large decision support systems, such as NED-2(Donald Nute ,2005, P.44-59), OntoWEDSS(Luigi Ceccaroni ,2003, P.785-797). But those systems are not automatic integration of model. This paper uses the idea of semantic Web services combining ontology, and puts forward the program-logic-combined model representation (PLC) method. Based on PLC representation, it gives a integrated system structure model with high expansibility, openness, automation and intelligent degree.

\section{PLC method}

Usually, there are some model representation method, such as program representation method, data representation method and logic representation method. Program representation method is easy to realize, but unfavorable to reuse model. Data representation method is favorable to realize automatic integration, but the degree of integrated intelligence is not high. Logic representation method supports reasoning, but is difficult to realize(Cai Shubin, 2009, P.713-719). In this paper the program-logic-combined model representation (PLC) method is proposed, which inherited the advantages of Program representation and logical representation, easy to implement and support reasoning. Also through the use of ontology model parameters, all kinds of model integration strategies can be designed based on the semantic matching, then create easy understanding integrated solutions. Programs using $\mathrm{C}++$, Java, $\mathrm{C}$ computer language can be easily to package and reuse. This makes the PLC method have higher openness and expansibility. The attributes of model public interface implementation class is explained through the corresponding Model Configuration Document (MCD). MCD is the logical representation of model and written by OWL, which is made up of 3 parts - model description, model parameter and model realization. This paper choice patent as the research object, because the range of patent field is very wide, so we take the "TV" subclass of patent field for example to introduce(Chen Guohai,2007, P.20-32).Then we give an ontology model of "TV" patent a description with OWL as follows.

$<$ owl:Class rdf:ID=" household_appliance " $>>$

$<$ owl:Class rdf:ID=" TV">

$<$ rdfs:label xml:lang="en" $>$ Television $</$ rdfs:label $>$

$<$ rdfs:subClassOf rdf:resource="\#household_appliance"/>

$<$ refs:comment rdf:datatype ="http://www.w3.org/2001/XMLSchema\#string"> This is a definition of TV patent. $<$ rdfs:comment $>$

$</$ owl:class $>$

$<$ owl:DatatypeProperty rdf:ID="patentName">

$<$ rdfs:domain rdf:resource="\# household_appliance "/>

$<$ rdfs:rangerdf:resource="http://www.w3.org/2001/XMLSchema\#string"/>

$<$ rdfs:comment rdf:datatype $=$ "http://www.w3.org/2001/XMLSchema\#string" $>$ Patent Name $</$ rdfs:comment $>$

$<$ owl:DatatypeProperty $>$

…...

$<$ owl:DatatypeProperty rdf: ID=" appNo">

$<$ rdfs:domain rdf:resource="\#household_appliance"/>

$<$ rdfs:range rdf:resource $="$ http://www.w3.org/2001/XMLSchema\#string"/ $>$

$<$ rdfs:comment rdf:datatype="http://www.w3.org/2001/XMLSchema\#string" $>$ appNo</rdfs:comment>

$</$ owl:DatatypeProperty $>$ 


\section{Presentation of model parameter}

In order to further decoupling model and data, this paper introduced ontology to present the meaning of data, then used semantic to connect model with data. Experts in the field and knowledge engineer, create the Domain Data Ontology of patent field (DAO), which definite semantic concepts of the field Data. The DAO used protege to design, OWL to descript. Figure 1 is the screenshot of DAO, which set the "TV" is a subclass of "household_appliance" class. The "TV" include 17 attributes, such as applicant, appNo, abstract, appDate, patentName(Chen Guohai,2007, P.20-32).Data Administrators describe data and use DAO to create data entity, semantic concepts and their corresponding relation.

Here is a definition about a class named "TV", whose superclass is "household_appliance". The "TV" include 17 attributes, such as applicant, appNo, abstract, appDate, patentName. Then we take the "The LCD televisions with computer functions" for example to give a description with OWL referenced by the PRC state intellectual property office sites (http://www.sipo.gov.cn/sipo2008/ zljs).

$<$ TV rdf:ID=" The LCD televisions with computer functions ">

$<$ International Publication xml:lang="en" $><$ International Publication $>$

$<$ Applicant xml:lang="en" $>$ Shenzhen Hasee Computer Company $<$ Applicant $>$

$<$ Publication Number xml:lang="en" $>$ CN200947641 < Publication Number $>$

$<$ International Application xml:lang="en" $><$ International Application $>$

$<$ Application Number xml:lang="en">200620136701.9</ Application Number $>$

$<$ Priority xml:lang="en" $><$ Priority $>$

$<$ Publication Date rdf:datatype=http://www.w3.org/2001/XMLScgema\#date $>2007.09 .12<$ Publication Date $>$

$<$ Full-text xml:lang="en">http://10.13.45.218/books/XX/200620136701.9/2151.tif $></$ Full-text $>$

$<$ Application Date rdf:datatype= "http://www.w3.org/2001/XMLScgema\#date" > 2006.09.19 < Application Date>

$<$ Attorney xml:lang="en" $><$ Attorney $>$

$<$ Agent xml:lang="en" $></$ Agent $>$

$<$ IPC xml:lang="en">H04N5/00(2006.01)I $<$ IPC $>$

$<$ Abstract xml:lang="en"> A LCD television with the integration function of TV and computer, including: TV ,computer parts, power and LCD part. Among them, the output of television parts, electrical connection LCD screen. TV, LCD, computer circuit board assembly in the box, saving space, reduce the cost and easy to control. $<$ Abstract $>$

$<$ Name xml:lang="en" $>$ The LCD televisions with computer functions $<$ Name $>$

$<$ Address xml:lang="en" $>518112$ Guangdong province shenzhen longgang industrial area $<$ / Address $>$

$<$ Inventor xml:lang="en" $>$ Cai Zhuo-xian $<$ Inventor $>$

\section{Structure of model integrated system}

In this section, structure of model integrated system is briefly introduced. As figure 2 (Cai Shu-bin, 2009, P.713-719)shows, the system is divided into two modules_ data and model. Data module concern data integration, data storage and retrieval. Model module concerns present management, operation and integration of model. Model integration strategy is an important part of the decision support solution. Decision support solutions include data, model and output area. Data area designated data. Model district designated the preferred integration strategy, and generating configuration document collection. The output area designated semantic concept needed by data.

\section{Summary and Discussion}

Model integration is an important content of the DSS, and former presentation of model parameters lack useful semantic information, so the effect of model integration is not ideal, integrated results is difficult to understand and refinement. This paper proposed a program-logic-combined model representation method based on ontology, which is not only easily realization, but also support in the comprehensive consideration of integration methods, the existing system, scalability and openness. Using of parameters information of the configuration document and semantic based on ontology to integrate model, this method is not only support various automatic and intelligent degree of integration strategy of model, also easy to understand, the integration of the modified 
refinement.

\section{References}

Borst P. and Akkermans H. (1997). An Ontology Approach to Product Disassembly. EKAW 1997, Spain :Sant Feliu de Guxols.P.15-19.

Cai Shu-bin, Ming Zhong, Li Shi-xian, Liu Xian-ming. (2009). Ontology based model integration. Chinese Journal of Electronics. No.37(4).P.713-719.

Chen Guohai. (2007). Research on Some Key Technologies of Enterprise Patent Management \& Analysis System.Master Thesis, Zhejiang university, Hang zhou.P.20-32.

Donald Nute, Walter D Potter, Zhiyuan Cheng, et al. (2005). A method for integrating multiple components in a decision support system. Computers and Electronics in Agriculture. No.49(1).P.44-59.

Gruber,T.R. (1993). A translation approach to portable ontologies. Knowledge Acquisition.No.5(2).P. 199-220.

H K Bhargava, R Krishnan, R Muller. (1997). Decision support on demand: emerging electronic markets for decision technologies . Decision Support Systems.No.19(3).P.193-214.

Kaushal Chari. Model composition in a distributed environment. (2003). Decision Support Systems.No.35(3).P.399-413.

Luigi Ceccaroni, Ulises Cortes, Miquel Sanchez-Marre. (2003). OntoWEDSS: augmenting environmental decision-support systems with ontologies. Envionmental Modeling \& Software.No.19(9).P.785-797.

M Holocher, R Michalski ,D Solte, F Vicula. (1997). MIDA: an open systems architecture for model2oriented integration of data and algorithms. Decision Support Systems.No.20(2).P.135-147.

O B Kwon. (2003). Meta web service: building web-based open decision support system based on web services. Expert Systems with Applications.No.24(4).P.375-389.

PRC State Intellectual Property Office Sites. [Online] Available:http://www.sipo.gov.cn/sipo2008/ zljs(November 28,2009)

Studer Rudi Riehard Benjamins, and Dieter Fensel. (1997). Knowledge Engineering: Principles and Methods. Data and Knowledge Engineering.No.25(1-2).P.161-197.

Uschold Mike, and Michael Gruninger. (1996). Ontologies: Principles Methods and Applications. Knowledge Engineering Review.No.11(2).P.93-136.

Web Ontology Language (OWL) Overview W3C Recommendation 10 February 2004. (http://www.w3.org/TR/2004/REC-owl-features-20040210)

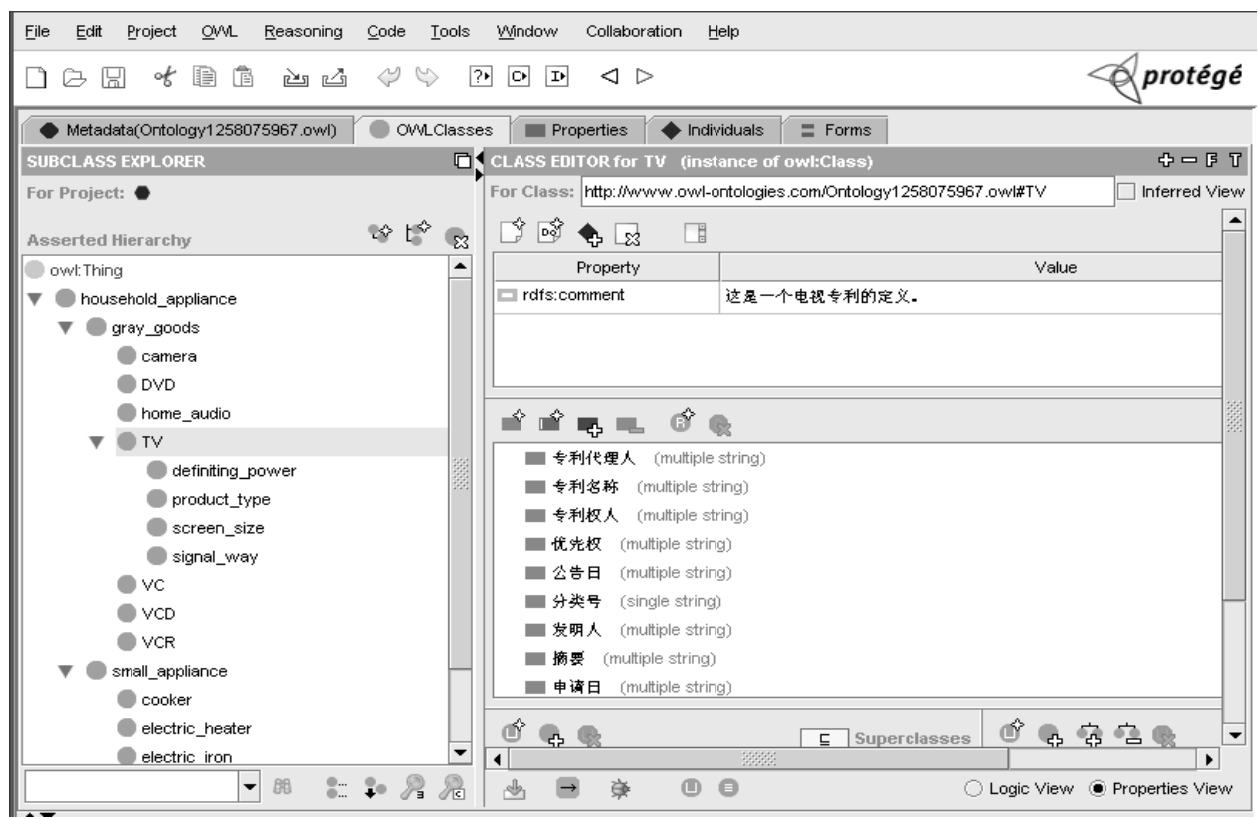

Figure 1. The Screenshot of "Household_appliance" Ontology 


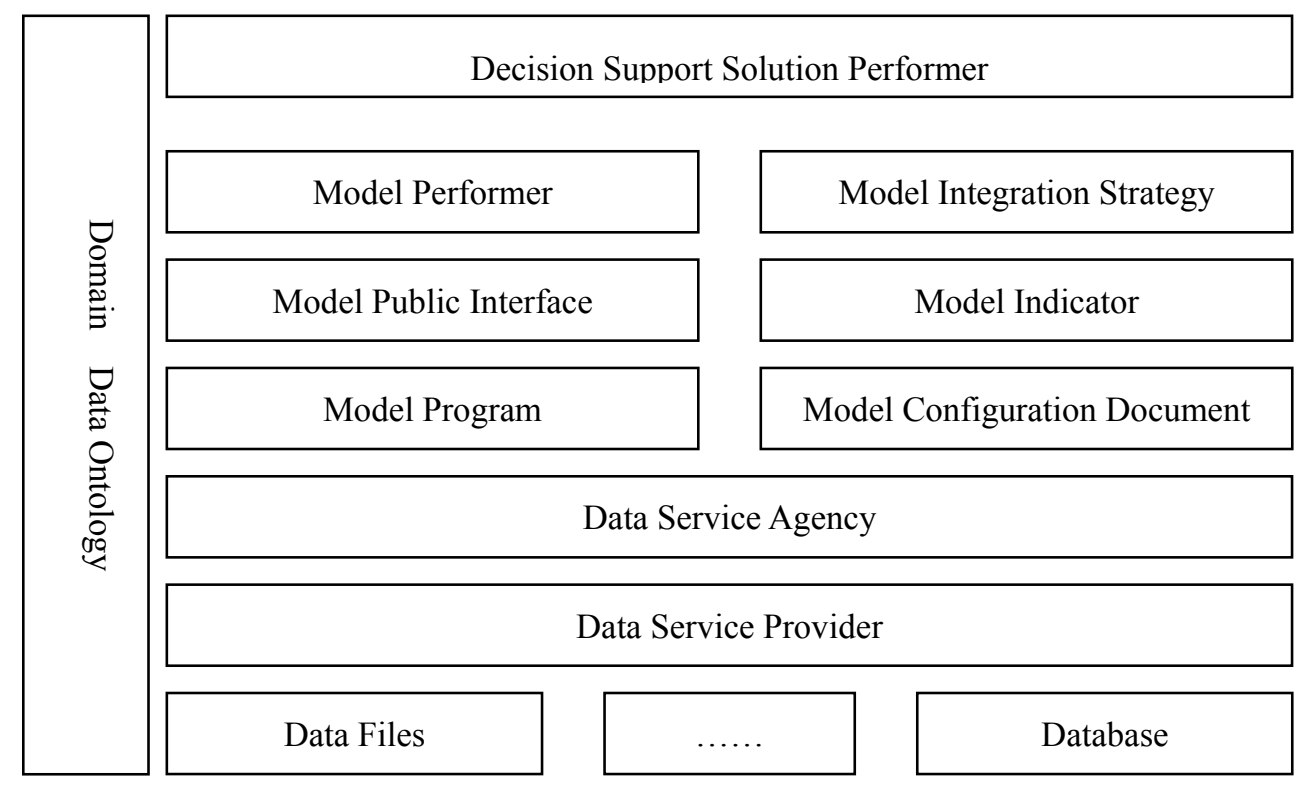

Figure 2. Structure of Model Integrated System 